\title{
Transforms in Optics: The Legacy of P.-M. Duffieux
}

Peter W. Hawkes

CEMES-LOE du CNRS, BP 4347, 31055 Toulouse Cedex 4, France

(Received October 24; accepted December 6, 1996)

PACS.02.90.+p - Other topics in mathematical methods in physics

PACS.41.85.-p - Beam optics

PACS.42.30.Kq - Fourier optics

PACS.02.30.Nw - Fourier analysis

PACS.02.30.Px - Abstract harmonic analysis

PACS.07.78.+s - Electron, positron, and ion microscopes, electron diffractometers, and related techniques

\begin{abstract}
The fiftieth anniversary of the publication of the first book on Fourier Optics, by P.-M. Duffieux, is recalled and the roles of the Fourier and many related transforms in image formation and image processing are recapitulated.
\end{abstract}

\section{Introduction}

It is just fifty years since the first book on what we now call "Fourier Optics" appeared. The title page of L'Intégrale de Fourier et ses Applications à l'Optique [1], privately printed for the author, Pierre-Michel Duffieux, by the Imprimeries Oberthur in Rennes, carries the date 1946, though Duffieux dates his Preface 1943 and the copyright date is 1947 . The optics community was slow to appreciate these ideas and it was only with the publication in 1959 of Principles of Optics [2] by Born and Wolf that they became widely known. Today, they have become part of the mainstream of optics, with extensive ramifications in image processing.

The Fourier transform is not, however, the only transform of interest in optics; the Karhunen-Loève and cosine transforms, the number-theoretic transforms, the Walsh, Haar and other transforms that have come to be associated with wavelet expansions are all encountered in the optical literature. Why are so many transforms in use and what purposes do they serve? There are the questions that we shall examine here.

\section{The Origin of the Integral Transforms}

All the familiar transforms based on continuous functions (circular functions or exponentials, Bessel functions, Legendre functions, ...) emerge when we solve a second-order linear homogeneous differential equation subject to boundary conditions. This differential equation typically has the form:

$$
\left(p y^{\prime}\right)-q y+\lambda \rho y=0
$$


in which the functions $p(x), q(x)$ and $\rho(x)$ are given and $\lambda$ is a parameter. The boundary conditions may be such that

$$
y(0)=y(X)=0
$$

or

$$
y^{\prime}(0)=y^{\prime}(X)=0
$$

for example. For many physical applications, it is important to realize that (1) is the Euler equation of a variational problem, namely,

$$
\delta \Lambda(y)=0
$$

subject to

$$
\int \rho y^{2} \mathrm{~d} x=1
$$

in which

$$
\Lambda(y)=\int\left(p y^{\prime 2}+q y^{2}\right) \mathrm{d} x
$$

and $\lambda$ emerges as the corresponding Lagrange multiplier. All solutions of (1) associated with different values of $\lambda$ are orthogonal, for consider the form of (1) for two such values, $\lambda_{i}$ and $\lambda_{j}$ :

$$
\begin{aligned}
\left(p y_{i}^{\prime}\right)^{\prime}-q y_{i}+\lambda_{i} \rho y_{i} & =0 \\
\left(p y_{j}^{\prime}\right)^{\prime}-q y_{j}+\lambda_{j} \rho y_{j} & =0 .
\end{aligned}
$$

On subtracting these and using the fact that the operator in (1) is Hermitian, it is clear that

$$
\left(\lambda_{i}-\lambda_{j}\right) \int \rho y_{i} y_{j} \mathrm{~d} x=0 .
$$

Even in the degenerate case, when several solutions correspond to the same value of $\lambda$, or eigenvalue, these solutions can be rendered orthogonal and we have thus constructed a set of eigenvalues and associated eigenfunctions. This set can be shown to be complete and we can hence expand any other function as a weighted sum or integral of the eigenfunctions.

There are thus as many "transforms" as equations of the form (1), but only a limited number of these possess properties of any interest. For a full discussion, see texts on the equations of mathematical physics, such as [3].

\section{Properties of Importance in Optics}

In the representation of the image-forming process, the most important property for a transform to possess is that it maps a convolution into a simpler kind of product. We shall see below that a very general consideration of linear shift-invariant systems leads directly to the Fourier transform but first we comment briefly on the transition from the continuous to the discrete Fourier transform. Although it is usual to study the continuous case first, any numerical calculation inevitably involves a set of discrete samples of the continuous functions and never the continuous functions themselves. For a discrete data set of the form $y(m), m=0,1, \ldots$, $N-1$, the continuous Fourier transform and its inverse become

$$
\begin{aligned}
\tilde{y}(k) & =\frac{1}{N} \sum_{m=0}^{N-1} y(m) W^{k m} \quad k=0,1, \ldots, N-1 \\
y(m) & =\sum \tilde{y}(k) W^{-k m}
\end{aligned}
$$


where

$$
W=\exp (-2 \pi i / N)
$$

and we note that

$$
\sum_{m} W^{k m} W^{-l m}= \begin{cases}N & \text { if } \frac{k-1}{N} \text { is an integer or zero } \\ 0 & \text { otherwise. }\end{cases}
$$

The (discrete) convolution $z(m)$ of two sequences $x(m)$ and $y(m)$ is defined by

$$
z(m)=\frac{1}{N} \sum_{h} x(h) y(m-h)
$$

and it is easy to show that

$$
\tilde{z}(k)=\tilde{x}(k) \tilde{y}(k) .
$$

Correlation is only trivially different. With the definition

$$
Z(m)=\frac{1}{N} \sum x(h) y(m+h)
$$

we have

$$
\tilde{Z}(m)=x^{*}(k) y(k) \text {. }
$$

Why is it that the Fourier transform has this special relation with convolution products? And are there other transforms that map such products into direct products?

The reason why the Fourier transform performs such a mapping can be understood by considering two kinds of matrices, Toeplitz matrices and circulant matrices. These are defined as follows. A matrix is said to be a Toeplitz matrix, $T$, if its elements $T(m, n)$ are such that

$$
T(m, n)=T(m-n) .
$$

The corresponding condition for a matrix to be a circulant matrix, $C$, is that

$$
C(m, n)=C(m-n) \text { modulo } N \text {. }
$$

Toeplitz matrices occur naturally in the representation of linear, shift-invariant systems, circulants occur in the study of linear periodic systems. They are thus the principal element in any convolutional formula, such as the relation between object and image. What are the eigenvalues and eigenvectors of such matrices? Whatever the values of the individual matrix elements $C(m, n)$, the eigenvectors of any circulant matrix are the same as the columns of the discrete Fourier transform matrix, $W^{k m}$. If we denote the latter by $F$, then for any circulant $C$

$$
F C F^{*}=\Lambda
$$

where $\Lambda$ is a diagonal matrix with the eigenvalues of $C$ along the diagonal. The latter are the discrete Fourier transform of the first column of $C$.

We thus see that we could have predicted that the Fourier transform would be the right one to use when confronted with a convolution. Are there any others? If so, why have they not emerged from the above argument? The reply to these two questions is that there are other transforms, or more exactly, there is a family of transforms that possesses the "convolution property" and that in fact, the existence of these transforms was latent in the reasoning that led to the Fourier transform but needed to be brought into the foreground. These transforms 
are the number-theoretic transforms (see [4] for the basic idea and [5] for a more general form) based on the finite fields $G F\left(p^{m}\right)$, the elements of which are ordered sets of $p$ digits. The total number of elements is $q=p^{m}$ and if $b$ is any primitive element of $G F\left(p^{m}\right)$, then

$$
b^{q-1}=1 \text { (modulo a certain polynomial) }
$$

and every nonzero element of the field can be expressed as a power of $b$. If we form a set of quantities

$$
\begin{aligned}
\lambda_{1} & =c_{0}+c_{q-2} b+c_{q-3} b^{2}+\ldots+c_{1} b^{q-2} \\
\lambda_{1} b & =c_{1}+c_{0} b+c_{q-2} b^{2}+\ldots+c_{2} b^{q-2} \\
\lambda_{1} b^{2} & =c_{2}+c_{1} b+c_{0} b^{2}+\ldots+c_{3} b^{q-2} \\
& \\
& \cdot \\
& \cdot \\
\lambda_{1} b^{q-2} & =c_{q-2}+c_{q-3} b+c_{q-4} b^{2}+\ldots+c_{0} b^{q-2}
\end{aligned}
$$

then

$$
\lambda \beta=C \beta
$$

where

$$
\beta^{\mathrm{T}}=\left(\begin{array}{lllll}
1 & b & b^{2} & \ldots & b^{q-2}
\end{array}\right)
$$

and

$$
C=\left(\begin{array}{ccccc}
c_{1} & c_{q-2} & c_{q-3} & \ldots & c_{1} \\
c_{1} & c_{0} & c_{q-2} & \ldots & c_{2} \\
c_{2} & c_{1} & c_{0} & \ldots & c_{3} \\
& \ldots & & & \\
c_{q-2} & \ldots & & & c_{0}
\end{array}\right)
$$

We can form $q-1$ sets of equations of this form by writing, for example,

$$
\lambda_{2}=c_{0}+c_{q-2} b^{2}+c_{q-3}\left(b^{2}\right)^{2}+\ldots c_{1}\left(b^{2}\right)^{q-2}
$$

or in general

$$
\lambda_{j} \beta^{(j)}=C \beta^{(j)}
$$

with

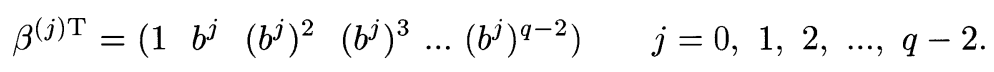

Collecting these together, we see that

$$
H C=\Lambda H
$$

in which $\Lambda$ is a diagonal matrix with the $\lambda_{j}$ along the diagonal and $H$ is a matrix with elements $h_{i j}$

$$
h_{i j}=b^{(q-1-i) j} .
$$

Thus the matrix $H$ again maps convolution products into direct products and as we can see, with hindsight, that we should have anticipated such a transform form our earlier reasoning. Both $b$ and the exponentials $W$ are roots of unity.

These number-theoretic transforms have the attraction that they are based on the integers. The rounding errors associated with the exponentials do not occur. They have the disadvantage that the field employed may need to be very large but work to circumvent this difficulty is continuing. 


\section{An Optimum Transform}

For any given task, it is natural to ask whether one transform is better than another. In threedimensional reconstruction, for example, the Fourier-Bessel transform is well adapted to a particular specimen symmetry. With the growing use of image databases [6], it is important to consider the most compact way of representing the vast amount of data in an image. Among the many types of coding that provide data compression, the transform codes have the advantage of simplicity, at the cost of discarding some information, regarded as dispensable. Which transform is best? More exactly, is there a transform that yields uncorrelated data? In most images, the grey levels of many adjoining pixels are highly correlated so that if only uncorrelated data are retained, this should be particularly efficient. The answer to this question has been known for many years but we draw attention to it briefly here. For full discussion see Section 5.1 in [7] or Section 71.4.1 in [8].

Given an image represented by a grey-level matrix $f$, with elements $f(m, n)$, we seek the set of matrices $\left\{\varphi^{(u, v)}\right\}$ such that

$$
f(m, n)=\sum_{u} \sum_{v} \varphi^{(u, v)}(m, n) F(u, v)
$$

and that

$$
\varepsilon\left\{F(u, v) F^{*}\left(u^{\prime}, v^{\prime}\right)\right\}=\varepsilon\{F(u, v)\} \varepsilon\left\{F^{*}\left(u^{\prime}, v^{\prime}\right)\right\}
$$

( $\varepsilon$ denotes expectation value). It can be shown that such a set does exist and the members of the set are the eigenmatrices (two-dimensional counterpart of eigenvectors) of the autocorrelation hypermatrix $R$ of the image $f$ :

$$
R(m, n, p, q)=\varepsilon\{f(m, n) f(p, q)\} .
$$

Thus

$$
\sum_{p} \sum_{q} R(m, n, p, q) \varphi^{(u, v)}(p, q)=\lambda_{u v} \phi^{(u, v)}(m, n)
$$

where the eigenvalues $\lambda_{u v}$ are given by

$$
\lambda_{u v}=\varepsilon\left\{|F(u, v)|^{2}\right\} .
$$

These complicated four- and two-dimensional structures can be considerably simplified by replacing the matrices by vectors and the hypermatrix by a matrix. The rows of the image $f$, for example, are placed one behind the other to form a long vector.

The equation (31) immediately reveals the major disadvantage of this transform, the Karhunen-Loève transform, namely that the transform matrix is not fixed, as in the case of the Fourier transform for example, but is different for every image. Fortunately, the performance of this transform is very nearly equalled by that of a transform that does have a fixed matrix, the so-called cosine transform [9], and it is that that would be used in practice. A convincing example of the efficiency of the Karhunen-Loève transform is to be found in [10].

\section{Multi-Resolution Transforms}

The Fourier transform and its close relatives (Bessel, Legendre, Gauss, Laguerre, ...) use information from the entire image in the calculation of each coefficient of the transform. This may be an important feature in the struggle against noise, as can be seen from the ptychographic methods of extracting phase information from STEM images developed by Rodenburg $[11,12]$. 


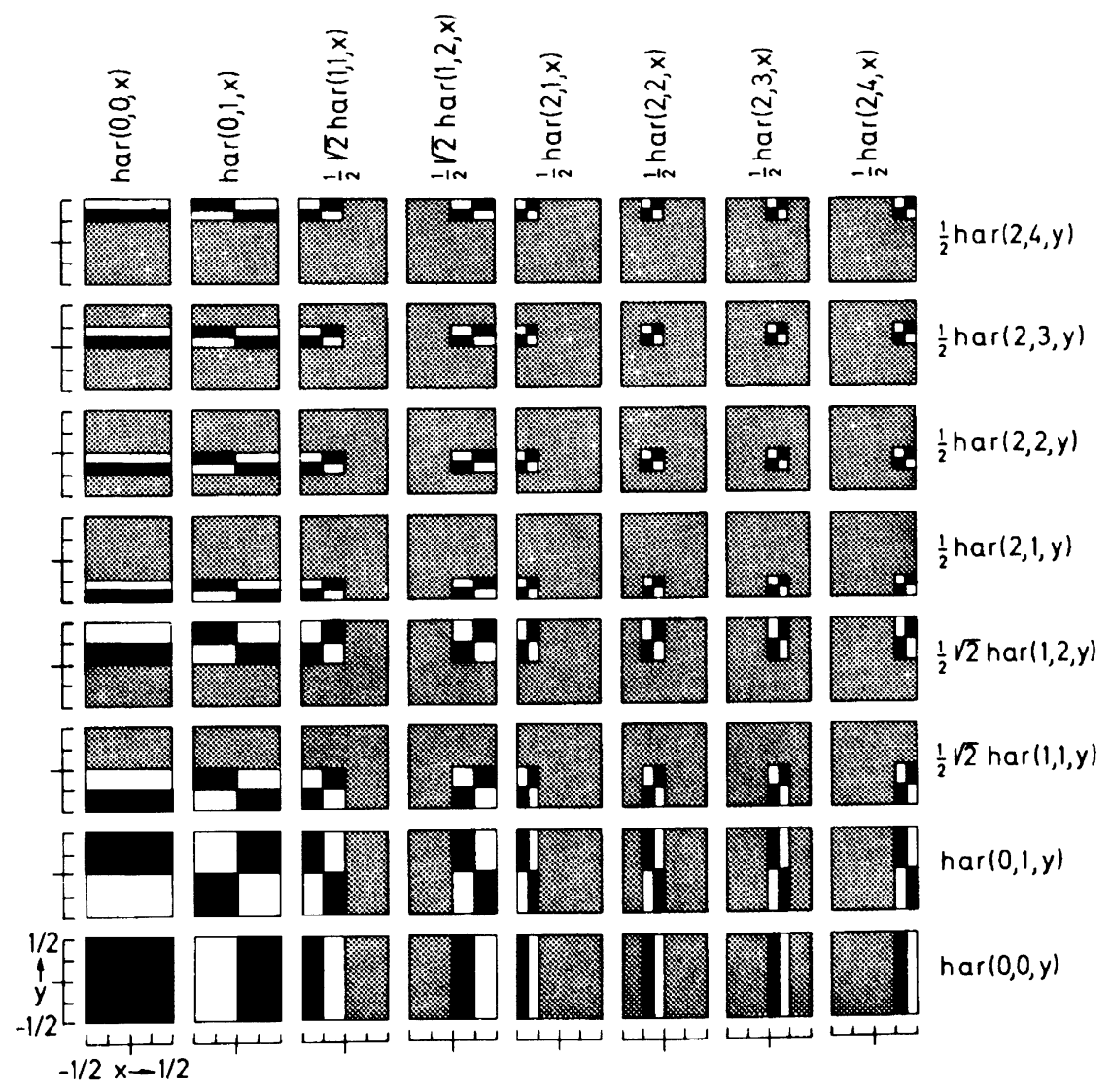

Fig. 1. - The Haar functions for a range of values of their labels and for values of their arguments $x, y$ in the intervals $-1 / 2<x<1 / 2,-1 / 2<y<1 / 2$. Black corresponds to 1 , grey to 0 and white to -1 .

It can, however, also be a drawback when the image is of uneven quality or interest. In such cases, transforms that analyse gradually smaller zones of the image become important. There are many such transforms; here we mention only the Haar transform, which illustrates the idea clearly. This transform is based on the (discrete or continuous) Haar functions, which form a periodic, orthonormal and complete set. They are defined over the interval $[0,1)$ by the expressions

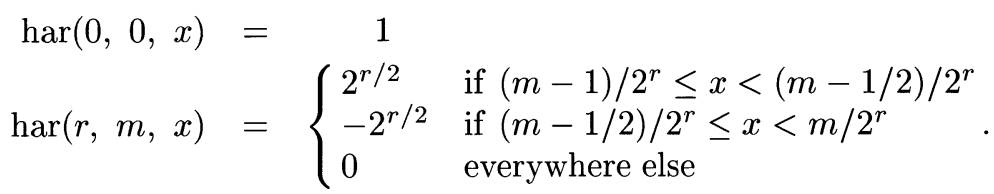

Figure 1 gives an idea of their appearance. The important point to notice is that, apart from the first two members, $\operatorname{har}(0,0, x)$ and $\operatorname{har}(0,1, x)$, they are zero over part of the interval $[0,1)$ and occupy less and less of the interval as we go up in order. A subset of the basis functions will thus contain information only about a zone within the interval, $[0,1 / 2)$ for example. 
The Haar transform is the archetypal member of a large group of such transforms, which have come to be known as wavelet transforms and have been intensively studied during the past decade [13]. In these, the basis functions of the transform are generated by dilatation and displacement of a "mother wavelet". If the latter is denoted by $\psi_{(0,0)}(n)$,

$$
\psi_{(0,0)}(n)=2^{-n / 2}, \quad n=0,1, \ldots, N-1
$$

then the daughter wavelets will be given by

$$
\psi_{m, k}(n)=2^{-m / 2} \psi\left(2^{-m} n-k\right), \quad m=1, \ldots, L ; \quad k=0,1, \ldots, 2^{L-m}-1 .
$$

The mother wavelet for the Haar transform, for example, is

$$
\psi= \begin{cases}1 & 0 \leq x \leq 1 / 2 \\ -1 & 1 / 2 \leq x<1 \\ 0 & \text { elsewhere }\end{cases}
$$

\section{Other Aspects}

The Fourier transform plays a central role in most branches of image processing. We consider the four broad areas of this subject in turn, namely acquisition and coding, enhancement, restoration and analysis.

\subsection{Acquisition and Coding}

We have already mentioned this above, in connection with the search for an optimal transform. The principle is simple and effective: the Fourier transform of an image is its spatial frequency spectrum. Low frequency components correspond to relatively coarse features of the image, higher frequencies correspond to fine detail or areas of the image in which the contrast is changing rapidly and irregularly; very high frequencies correspond to noise. Transform coding consists in truncating the transform beyond the point at which the system is capable of furnishing useful information.

\subsection{Enhancement}

Among the various methods of enhancing the quality of an image, we concentrate here on the (linear) convolutional methods and the (nonlinear) morphological techniques.

The first category contains the very extensive group of operations in which the grey level at each pixel is replaced by a weighted sum of the grey levels inside a window centred on the pixel in question. The simplest example, smoothing to reduce certain kinds of noise, consists in replacing the grey level by the mean value of the grey levels in the window. Since the window is moved systematically over the whole image, the operation may be regarded as a convolution of the image with the window function, whence the term "convolutional methods". Although it is tempting to invoke the convolution theorem, it is in practice not advantageous to calculate the Fourier transforms and form the direct product for the small windows (typically $3 \times 3$ or $5 \times 5$ ) that are encountered in convolutional filtering.

The other category, the morphological methods, also involves a kind of generalized convolution but in this case, the operations are intrinsically nonlinear. Whereas convolutions essentially involve summing over a set of products (e.g. Eq. (11)), the basic morphological operation (dilation) involves taking the maximum over a set of sums. Interest in mathematical 
morphology has grown enormously during the past decade and our understanding of the underlying mathematical structure has improved beyond measure. Nevertheless, it is only since 1993 that the existence of a transform that maps the basic morphological operation into a simpler "product", just as the Fourier transform maps the convolution product into a direct product, has been known [14-19]. Furthermore, this is no mere abstract development awaiting applications. Dorst and van den Boomgaard [17] show that image formation in near-field microscopes is intimately related to this new transform; an article by Bonnet et al. [20] is also relevant here. Suppose that $D(x)$ denotes the dilation of a signal $x$ by a structuring element $g$

$$
D(x)=x \oplus g .
$$

Then the lines

$$
x(z)=a z+b
$$

are the eigenfunctions of the operator $D$ because

$$
\begin{aligned}
D(a z+b) & =\bigvee_{\zeta} a(z-\zeta)+b+g(\zeta) \\
& =a z+b+G(a)
\end{aligned}
$$

where

$$
G(a)=\bigvee_{\zeta} g(z)-a z
$$

is a kind of eigenvalue (recall that $\max (\bigvee)$ and addition $(+)$ in morphology replace sum $\left(\sum\right)$ and product $(x)$ in traditional convolutions). Further developments of these ideas can be expected.

\subsection{Restoration}

Among the themes of restoration of greatest interest for electron imaging are three-dimensional reconstruction and the phase problem. The Fourier transform is an essential tool in 3-D reconstruction and indeed, one of the early wrangles between different schools of thought concerned the relative advantages of performing the reconstruction in direct space and in Fourier space. We refer to extended texts on the subject for further comment [21].

The phase problem, although now well understood, is far from solved, for real specimens at least. The problem is simply stated: high-resolution specimen information in an electron microscope is conveyed to the image as variations in the phase of the electron wavefunction but the detector responds not to the phase but to the amplitude. How can we recover the phase and, no less difficult a problem, how can we deduce the specimen structure from a knowledge of the phase distribution?

Both questions have a large literature, see Section 74 in [7] for a survey up to 1994 and [22,23] for later contributions. Here we merely wish to remind the reader that the Fourier transform, and especially the notions of Fourier optics that go back to Duffieux, play a central role in the Gerchberg-Saxton solution to the problem. The essence of their algorithm is to be found in the fact that diffraction pattern and image can be recorded separately in an electron microscope, giving two intensity patterns, but the (complex) wavefunctions to which these intensity patterns correspond are related by a Fourier transform. 


\subsection{Representation}

A unified representation of the innumerable algorithms that make up image processing is provided by "image algebra", a convenient mathematical structure in which the fundamental element is the image, defined very generally and the operations are the familiar addition, multiplication and maximum, together with their natural inverses subtraction, division and minimum. In terms of these, all the material that we have mentioned above can be easily represented, as can that of image analysis though less straightforwardly. For an introduction to this, see Section 70.2 in [7] and [24-27]. We mention it here because the Fourier transform conforms very naturally to one of the image types considered. The notion of image in image algebra is broad enough to encompass black-and-white (binary) images, grey-level images, coloured images, vector images (in which a set of values, the energy-loss spectrum for example, is associated with each pixel), multiple images (for which several images are associated with each pixel, corresponding to different detectors in a SEM, for example) and templates, the case we consider here. A template is an image-valued image, in which an entire image is associated with each pixel; such images arise naturally in the STEM for example [28] or in imaging systems that cannot be regarded as isoplanatic.

In the algebra, an image of any kind is regarded as a set. If $\mathbf{a}$ is an image, then

$$
\mathbf{a}=\{(\mathbf{x}, \mathbf{a}(\mathbf{x})) \mid \mathbf{x} \in \mathbf{X}\}
$$

where $\mathbf{x}$ is an address and $\mathbf{a}(\mathbf{x})$ is the value of the image at that address. For an ordinary grey-level image, $\mathbf{x}$ will be the pixel coordinates and $\mathbf{a}(\mathbf{x})$ the grey level at each pixel. For a vector image, $\mathbf{a}(\mathbf{x})$ might be the energy-loss spectrum. For a template, however, $\mathbf{a}(\mathbf{x})$ is itself an image and so for a template $\mathbf{t}$, we have

$$
\begin{array}{r}
\mathbf{t}=\{(\mathbf{x}, \mathbf{t}(\mathbf{x}) \mid \ldots\} \\
\mathbf{t}(\mathbf{x})=\left\{\left(\mathbf{y}, \mathbf{t}_{\mathbf{x}}(\mathbf{y}) \mid \ldots\right\}\right.
\end{array}
$$

in which we have written $\mathbf{t}(\mathbf{x}) \equiv \mathbf{t}_{\mathbf{x}}$ to avoid having to write $(\mathbf{t}(\mathbf{x}))(\mathbf{y})$. The most important operations between simple images and templates are "generalized" convolutions:

$$
\begin{aligned}
& \mathbf{b}=\mathbf{a} \oplus \mathbf{t}=\left\{(\mathbf{y}, \mathbf{b}(\mathbf{y}) \mid \mathbf{b}(\mathbf{y}))=\sum_{\mathbf{x}} \mathbf{a}(\mathbf{x}) \mathbf{t}_{\mathbf{y}}(\mathbf{x})\right\} \\
& \mathbf{b}=\mathbf{a} \odot \mathbf{t}=\left\{(\mathbf{y}, \mathbf{b}(\mathbf{y}) \mid \mathbf{b}(\mathbf{y}))=\bigvee \mathbf{a}(\mathbf{x}) \mathbf{t}_{\mathbf{y}}(\mathbf{x})\right\} .
\end{aligned}
$$

The first of these enables us to express all the convolutional filters compactly and the second corresponds to a basic morphological operation.

Consider now the Fourier transform $A(j, k)$ of an image $a(m, n)$ :

$$
A(j, k)=\sum \sum a(m, n) \exp \left\{-\frac{2 \pi i}{N}(j m+k n)\right\} .
$$

(This is the usual definition in image algebra.) If we regard the exponential as a template, $\mathbf{F}$, with

$$
\mathbf{F}_{(j, k)}(m, n)=\exp \left\{-\frac{2 \pi i}{N}(j m+k n)\right\}
$$


then the Fourier transform takes the form of a generalized convolution:

$$
\mathbf{A}=\mathbf{a} \oplus \mathbf{F} .
$$

For very full discussion of this representation see [29].

\section{Concluding Remarks}

These few examples give some idea of the richness of the many integral and discrete transforms that find a place in optics and in image processing. The recognition by Duffieux of the role of the Fourier transform, and by extension of other such transforms, has had very far-reaching repercussions that go far beyond the limits of optics, as the huge literature on algorithms of the Gerchberg-Saxton type bears witness. The place of Duffieux in the history of our subject is indeed secure.

\section{References}

[1] Duffieux P.-M., L'Intégrale de Fourier et ses Applications à l'Optique (privately published, 1946).

[2] Born M. and Wolf E., Principles of Optics (Pergamon, Oxford 1959).

[3] Jeffreys H. and Jeffreys B., Methods of Mathematical Physics (Cambridge University Press, Cambridge 1956).

[4] Arambepola B. and Rayner P.J.W., IEEE Trans. A SSP-28 (1980) 407.

[5] Hawkes P.W., Optik 42 (1975) 433.

[6] Marabini R., Vaquerizo C., Fernández J.J., Carazo J.M., Engel A. and Frank J., J. Struct. Biol. 116 (1996) 161.

[7] Rosenfeld A. and Kak A.C., Digital Picture Processing, vol. 1 (Academic Press, New York 1982).

[8] Hawkes P.W. and Kasper E., Principles of Electron Optics, vol. 3 (Academic Press, London 1994).

[9] Rao K.R. and Yip P., Discrete Cosine Transforms (Academic Press, Boston 1990).

[10] Burge R.E., Browne M.T., Charalambous P., Clark A. and Wu J.K., J. Microscopy 127 (1982) 47.

[11] Rodenberg J.M., in "Proc. 11th Eur. Cong. on Electron Microscopy", Dublin (1996) to be published.

[12] Nellist P.D., McCallum B.C. and Rodenburg J.M., Nature 374 (1995) 630.

[13] Aldroubi A. and Unser M., Wavelets in Medicine and Biology (CRC Press, Boca Raton 1996).

[14] Maragos P., in "Proc. Int. Workshop on Mathematical Morphology and its Applications to Signal Processing", Barcelona (1993), p. 168.

[15] Dorst L. and Boomgaard R. van den, in "Proc. Int. Workshop on Mathematical Morphology and its Applications to Signal Processing", Barcelona (1993), p. 245.

[16] Maragos P., Signal Proc. 38 (1994) 57.

[17] Dorst L. and Boomgaard, R. van den, Signal Proc. 38 (1994) 79.

[18] Maragos P., IEEE Trans. Signal Proc. 43 (1995) 864.

[19] Maragos P., in "Mathematical Morphology and its Applications to Image Processing", Fontainebleau 1994 (Kluwer, Dordrecht, 1994) p. 149. 
[20] Bonnet N., Dongmo S., Vautrot P. and Troyon M., Microsc. Microanal. Microstruct. 5 (1994) 477.

[21] Frank J., Three-dimensional Electron Microscopy of Macromolecular Assemblies (Academic Press, San Diego 1996).

[22] P.W. Hawkes, J. Frank and W.O. Saxton, Eds, Signal and Image Processing in Electron Microscopy, Scanning Microsc. Suppl. 11 (1997).

[23] K. van der Mast, Guest Ed., Ultramicroscopy 64 (1996).

[24] Ritter G.X., Wilson J.N. and Davidson J.L., Computer Vision Graph. Image Proc. 49 (1990) 297.

[25] Ritter G.X., Adv. Electron Electron Phys. 80 (1991) 243.

[26] Ritter G.X. and Wilson J.N., Handbook of Computer Vision Algorithms in Image Algebra (CRC Press, Boca Raton 1996).

[27] Hawkes P.W., Microsc. Microanal. Microstruct 6 (1995) 159-177.

[28] Hawkes P.W., in "Proc. 13th Int. Conf. on Electron Microscopy, Paris", vol. 1 (1994) p. 495.

[29] Ritter G.X. and Gader P.D., J. Parallel Distrib. Comput. 4 (1987) 7. 